http://dx.doi.org/10.12775/szhf.2020.014

\author{
Alexei Zhavoronkov \\ Institut für Philosophie, Russian Academy of Sciences, \\ Moskow, RUSSIAN FEDERATION
}

Immanuel Kant Baltic Federal University, Kaliningrad, Russian Federation

E-MAIL: ALAUDARIUS@GMAIL.COM

ORCID: 0000-0001-8362-1782

\title{
Die soziologische Rezeption von Kants Anthropologie im 20. Jahrhundert: Probleme und Beispiele
}

Die in den letzten anderthalb Jahrzehnten rasch anwachsende Literatur zu Kants Anthropologie bezeugt, dass diese Seite seiner Philosophie - trotz aller Kritik an einzelnen Aspekten - nicht mehr als eine bloße Ansammlung von zeitgenössischen Vorurteilen, als ein zugleich theoretisch-wissenschaftlich und empirisch mangelhaftes Unternehmen oder als ein einsam dastehendes Kuriosum des Kantischen Denkens betrachtet wird. Diese Entwicklung bringt auch die Frage mit sich nach der Aktualität von Kants anthropologischem Projekt für gegenwärtige Debatten in philosophischer Anthropologie, Philosophie der Biologie, Ethik, sozialer und politischer Philosophie. Einen wesentlichen Teil dieses Problemkreises bildet das Thema von Kants tatsächlichem und potentiellem Einfluss auf die modernen Sozialwissenschaften wie Politische Theorie oder Soziologie. Mit anderen Worten, um die spezifischen Voraussetzungen der Einbeziehung von Kants Anthropologie in aktuelle 
Debatten auszuloten, sollten wir zuerst verstehen, welche Elemente von Kants Anthropologie für die bisherige Rezeption relevant waren. Im vorliegenden Artikel, der sich diesem letzteren Thema widmet, werde ich die soziologische Seite von Kants Anthropologie beschreiben sowie die Haupteigenschaften ihrer Rezeption in der deutsch- und englischsprachigen Soziologie des 20. Jahrhunderts an einigen Beispielen illustrieren.

\section{Die soziologische Dimension von Kants Anthropologie}

Die Beantwortung der Frage nach sozialen Aspekten von Kants Anthropologie, die die englisch- und deutschsprachige Soziologie beeinflusst haben, ist ihrerseits nicht ohne eine adäquate Vorstellung der Genese, Struktur und Entwicklung seines anthropologischen Projekts sinnvoll möglich. Einzelne anthropologische Ideen und Begriffe finden wir bereits in Kants Schriften der vorkritischen Periode, v. a. in Beobachtungen über das Gefühl des Schönen und Erhabenen (1764), im Versuch über die Krankheiten des Kopfes (1764) und in Von den verschiedenen Rassen der Menschen (1775). Als eigene Disziplin aber entfaltet sich Kants ,pragmatische' Anthropologie, deren frühe Konturen sich in Vorlesungen und Briefen der 1770er Jahre finden ${ }^{1}$, erst während der kritischen Periode zum festen Bestandteil seines Denkens. Neben der Anthropologie in pragmatischer Hinsicht (1798) sind v.a. Beantwortung der Frage: Was ist Aufklärung? (1784), Bestimmung des Begriffs einer Menschen-

${ }^{1}$ Obwohl Kants Vorlesungen über Anthropologie, die den 25. Band der Gesammelten Schriften ausmachen, nur als von Kant nicht autorisierte Nachschriften seiner Schüler existieren und in mehreren Fällen eine Kompilation von Vorlesungen aus verschiedenen Semestern darstellen, bieten sie eine einzigartige Möglichkeit, frühe Entwicklungsstufen von mehreren anthropologischen Schlüsselthesen und Begriffen Kants (wie etwa 'ungesellige Geselligkeit') zu beobachten. Mehr zur Entstehung und zum Inhalt der Nachschriften-Manuskripte s. in Reinhardt Brandt, Werner Stark, "Einleitung", in: Immanuel Kant, Gesammelte Schriften. Akademie-Ausgabe. Bd. XXV: Vorlesungen über Anthropologie (Berlin: De Gruyter, 1997), VII-CLI. Für einen zusammenfassende Charakteristik der Geschichte der Gesammelten Schriften, darunter auch der editorischen Situation mit Kants Vorlesungen, s. Werner Stark, “'Diese Dinge' und andere Varia”, in: Zustand und Zukunft der Akademie-Ausgabe von Kant's gesammelten Schriften, Kant-Studien, Bd. 91, Sonderheft, ed. R. Brandt, W. Stark (Berlin: De Gruyter, 2000), 1-16. 
race (1785), Mutmaßlicher Anfang der Menschengeschichte (1786) und Über den Gebrauch teleologischer Principien in der Philosophie (1788) als anthropologische Schlüsselquellen zu erwähnen. Anthropologische Thesen finden wir aber auch in Kants drei Kritiken sowie im Traktat Zum ewigen Frieden.

In seinen Schriften entwickelt Kant eine neuartige, in der bisherigen Forschung nur selten thematisierte Konzeption von , pragmatischer' Handlungsrationalität ${ }^{2}$. In der Anthropologie in pragmatischer Hinsicht (1798) spielt die pragmatische Vernunft eine besondere Rolle: Will man den Menschen „in pragmatischer Hinsicht“ systematisch erkennen - und zwar über seinen Charakter als Naturwesen hinaus -, so muss man ihn als zwar nicht vollkommen vernünftiges, aber doch vernunftfähiges Wesen (animal rationabile $e^{3}$ ) begreifen - und die anthropologische Erkenntnis darauf richten, was der Mensch „als frei handelndes Wesen aus sich selber macht, oder machen

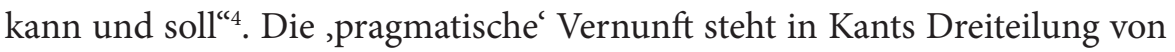
praktischen Imperativen zwischen rein ,technischen', durch kausale Naturgesetze bedingte Vorschriften einerseits und rein moralischen oder kategorischen Imperativen der reinen praktischen Vernunft andererseits. Was "pragmatische Imperative“ genau gebieten, reicht bei Kant von „Ratschlägen“ zur Verfolgung des nur individuellen, eigenen Glücks bis zu Empfehlungen darüber, wie die Menschheit als Ganzes die von der Moral gesetzten Normen und Ziele realisieren kann ${ }^{6}$. Jedenfalls geht diejenige pragmatische Vernunft, die in der Anthropologie vermittelt wird, über eine bloß instrumentelle oder technische Rationalität hinaus, indem sie den Umstand berücksichtigt, dass wir Menschen einander schon faktisch - nicht nur aus moralischen Prinzi-

${ }^{2}$ Ich bedanke mich bei Prof. Dr. Thomas Sturm und Dr. Alexey Salikov für die Möglichkeit, diesen äußerst wichtigen und in der Forschung eher wenig beachteten Begriff Kantsmiteinander zu besprechen, sowie für ihre Ratschläge und Hilfe bei unserer Arbeit am gemeinsamen Projekt zu Kants Anthropologie und Sozialwissenschaften, dessen Teil mein Thema zu Kants Einfluss auf die Soziologie ausmacht.

${ }^{3}$ Immanuel Kant, Anthropologie in pragmatischer Hinsicht (weiter - ApH), in: Akademie-Ausgabe (weiter - AA), Bd. VII, 321.

${ }^{4} \mathrm{Kant}, \mathrm{ApH}$, in: AA, Bd. VII, 119.

${ }^{5}$ Vgl. Immanuel Kant, Grundlegung zur Metaphysik der Sitten, in: AA, Bd. IV, 414-419; ApH, in: AA, Bd. VII, 322; Vorlesungen über Anthropologie (weiter - VA), in: AA, Bd. XXV, 855.

${ }^{6}$ Idem, ApH, in: AA, Bd. VII, 329. 
pien heraus, wie Kant es bekanntlich auch meint - nicht als bloße Mittel für die je eigenen Zwecke gebrauchen können. Vielmehr müssen wir, wenn wir über angemessene pragmatische „Weltkenntniß $3^{\text {“7 }}$ oder „Weltklugheit" ${ }^{\text {“8 }}$ verfügen oder verfügen wollen, uns gegenseitig als Wesen erkennen, die in Abhängigkeiten voneinander stehen, die durch die Erfordernisse der Erziehung und Anerkennung bestimmt sind, die gegebene gesellschaftliche Handlungsnormen stets modifizieren oder neu erfinden können, also geteilte Regeln selbst erzeugen, und damit die gesellschaftliche Realität selbst aktiv miteinander gestalten, in der sie dann leben - und damit einander letztlich als ,Weltbürger' verstehen. Die pragmatische Vernunft oder,Weltklugheit' gebietet daher, die Perspektiven anderer rationaler Akteure in das eigene Handeln und die sie leitenden sozialen Regeln einzubeziehen - und sich selbst zu einer zunehmend rationaleren Person auszubilden, die sich entsprechend in der menschlichen Gesellschaft verhält und diese auch mitzugestalten versucht ${ }^{9}$.

In der Konzeption von Kants pragmatischer Anthropologie finden sich sowohl methodologische als auch inhaltliche Verbindungen zu Elementen seiner kritischen Philosophie, die ihren Ausdruck in den drei Kritiken und in den anderen Schriften der 1780er und der 1790er Jahre finden. So können wir etwa von - freilich nicht unproblematischen - Zusammenhängen zwischen der Anthropologie in pragmatischer Hinsicht (ApH), der Selbstbewusstseinstheorie aus der ersten $K_{r i t i k}{ }^{10}$, der Freiheitslehre aus den ersten beiden Kritiken und der Organismustheorie aus der dritten Kritik sprechen ${ }^{11}$. Auch gibt es wichtige Verbindungen hinsichtlich der systematischen Struktur der pragmatischen Anthropologie und Kants kritischem, auch historisch

${ }^{7}$ Ibidem, 120.

${ }^{8}$ Kant, Grundlegung zur Metaphysik der Sitten, 416-417.

${ }^{9}$ Mehr dazu in Thomas Sturm, Kant und die Wissenschaften vom Menschen (Paderborn: Mentis Verlag, 2009), 501.

${ }^{10} \mathrm{~S}$. Thomas Sturm, „Reines und empirisches Bewusstsein in Kants Anthropologie: Das 'Ich' und die rationale Charakterentwicklung", in: Immanuel Kant - Die Einheit des Bewusstseins, ed. G. Motta, U. Thiel (Berlin/Boston: De Gruyter, 2017), 195-220.

${ }^{11}$ Vgl. Robert B. Louden, Kant's Impure Ethics: From Rational Beings to Human Beings (New York: Oxford University Press, 2000); Allen Wood, "Kant and the Problem of Human Nature", in: Essays on Kant's Anthropology, ed. B. Jacobs, P. Kain (Cambridge: Cambridge University Press, 2003), 38-59. 
neuartigem Begriff der Wissenschaft ${ }^{12}$. Dass Kant während der kritischen Periode seine Position zu anthropologischen Schlüsselthemen stufenweise ändert, zeigt schließlich auch die Transformation seines Begriffes der Rasse von einem eurozentrischen, moralisch diskriminierenden Konzept in den 1770er und 1780er Jahren zu einer kosmopolitischen und moralisch neutralen Idee in den 1790er Jahren ${ }^{13}$.

Obwohl viele der Grundlagen und Inhalte von Kants Anthropologie inzwischen gründlich erforscht sind, gilt dies nicht für ihre spezifischen sozialen Gehalte. Diese Thematik ist zwar in den letzten 15 Jahren allmählich von der philosophischen Forschung aufgegriffen worden ${ }^{14}$, es gibt jedoch noch keine systematische Studie, die alle Schlüsselaspekte dieses Problemkomplexes behandelt. Gleich der ethischen und politischen Seite von Kants Anthropologie, manifestiert sich auch ihre soziologische Dimension sowohl in verschiedenen kleinen und großen Schriften als auch in den Vorlesungen zur physischen Geographie und zur Anthropologie, etwa am Beispiel seiner Konzeption der , ungeselligen Geselligkeit ${ }^{15}$, des Konzepts der sozialen Natur des Bösen und des natürlichen Hangs zum ‘bösen' Handeln und der Idee einer weltbürgerlichen Gesellschaft. Die Idee der , ungeselligen Geselligkeit' ist ein zentrales Element von Kants pragmatischem Ansatz: Wir Menschen sind einerseits von egoistischen Motiven getrieben, aber ebensosehr auch in Erziehung, dem Streben nach Anerkennung und anderem mehr auf die Gesellschaft zwingend angewiesen. Wer wirkliche, Weltklugheit' oder pragmatische

${ }^{12}$ Vgl. Sturm, Kant und die Wissenschaften vom Menschen, 499.

${ }^{13}$ Mehr dazu etwa in Alexey Zhavoronkov, Alexey Salikov, “The Concept of Race in Kant's Lectures on Anthropology", Con-Textos Kantianos 7 (2018): 275-292 (am Beispiel von Kants anthropologischen Vorlesungen).

${ }^{14}$ S. bereits Patrick J. Frierson, Freedom and Anthropology in Kant's Moral Philosophy (Cambridge: Cambridge University Press, 2003), 142-145 et passim. S. auch Holly J. Wilson, Kant's Pragmatic Anthropology: Its Origin, Meaning and Critic Significance (New York: SUNY Press, 2006), 62-69 et passim; Günter Zöller, "Kant's Political Anthropology", Kant Yearbook 3 (2011): 131-161; Philip J. Rossi, "Cosmopolitanism - Kant's Social Anthropology of Hope", in: Kant und die Philosophie in weltbürgerlicher Absicht: Akten des XI. Kant-Kongresses 2010, Bd. 4, ed. S. Bacin, A. Ferrarin, C. La Rocca, M. Ruffing (Berlin: De Gruyter, 2013), 827-837; Paul Guyer, "Play and Society in Kant's Anthropology", in: Reading Kant's Lectures, ed. R. R. Clewis (Berlin/Boston: De Gruyter, 2015), 223-241.

${ }^{15}$ An einigen Stellen, etwa in Kants Idee zur allgemeinen Geschichte in weltbürgerlicher Absicht (AA, Bd. VIII, 24), wird sie auch als ,Ungeselligkeit' bezeichnet. 
Vernunft erlangen will, muss diese Spannung erkennen und mit ihr umzugehen lernen. Die Natur hat laut Kant „den Keim der Zwietracht“ in die Menschengattung gelegt, sie aber zugleich zur Sozialität bestimmt und also offenbar "gewollt“, dass die „Vernunft aus dieser [Zwietracht] Eintracht, wenigstens die beständige Annäherung zu derselben, herausbringe “16. Das, was der Mensch „aus sich macht, oder machen kann und soll ${ }^{\text {“17 }}$, ist vor dem Hintergrund der Dynamik der sozialen Interaktion bzw. des sozialen Wettbewerbs zu verstehen ${ }^{18}$. Als „empirische Wissenschaft vom Menschen als frei handelndem Wesen "19 schlägt die Anthropologie eine Brücke von Kants Überlegungen zur menschlichen "Charakteristik“" (s. Teil 2 der Anthropologie in pragmatischer Hinsicht) zu seiner Idee der menschlichen Freiheit, die v.a. in den drei Kritiken behandelt wird ${ }^{20}$. Seine Vorstellung von gesellschaftlichen Verhältnissen im Lichte seines Konzeptes der sozialen Natur des Bösen und des natürlichen Hangs zum 'bösen' Handeln ${ }^{21}$ lässt sich als ein Kompromiss bzw. eine Mittelposition zwischen dem anthropologischen Pessimismus von Hobbes und dem sozial-politischen Optimismus Rousseaus verstehen. Die Idee einer weltbürgerlichen Gesellschaft, die als Antwort auf die Herausforderung durch die , ungesellige Geselligkeit' des Menschen zu verstehen ist, wird ihrerseits in der ApH als „ein regulatives Prinzip [der] Bestimmung des Menschengeschlechts" verstanden ${ }^{22}$. Auf allgemein-methodologischer Ebene steht die moderne Soziologie mit ihrem holistischen Ansatz, der sowohl kulturelle, politische und ökonomische als auch individual-ethische Aspekte des menschlichen Lebens mit einbezieht, Kants Konzept der pragmatischen Anthropologie als ,Weltkenntnis' nahe, das praktische Anwendbarkeit zum Ziel hat und mit einer spezifischen, diesem Ziel dienenden Idee der Klugheit operiert $^{23}$.

${ }^{16}$ Kant, ApH, in: AA, Bd. VII, 322.

${ }^{17}$ Ibidem, 119.

${ }^{18}$ Vgl. Sturm, „Reines und empirisches Bewusstsein in Kants Anthropologie“, 217.

${ }^{19}$ S. Sturm, Kant und die Wissenschaften vom Menschen, 475.

${ }^{20}$ S. dazu insbes. Wood, „Kant and the Problem of Human Nature“, 42-45.

${ }^{21}$ Vgl. I. Kant, VA, in: AA, Bd. XXV, 930 et passim.

${ }^{22} \mathrm{Kant}, \mathrm{ApH}$, in: AA, Bd. VII, 331.

${ }^{23}$ Vgl. etwa Allen Wood, "Unsocial Sociability: The Anthropological Basis of Kantian Ethics”, Philosophical Topics 19 (1991): 325-351. 
Die Grundlagen der modernen Sozialwissenschaften wurden bekanntlich während der Aufklärung gelegt, als sich die Idee durchsetzte, dass die Welt durch den Gebrauch der Vernunft und empirische Untersuchung erkennbar sei. Indem es aber bereits eine umfassende Forschungsliteratur über Denker wie Hume, Rousseau, Ferguson, Adam Smith und Herder, die die Entwicklungen der Sozialwissenschaften beeinflusst haben, besteht eine erhebliche Lücke hinsichtlich Kants. Dies ist umso erstaunlicher, weil Kants Konzeption sich wesentlich von denen seiner Zeitgenossen unterscheidet. Seine pragmatische Anthropologie hielt er für eine empirische akademische Disziplin, die den Studenten wichtige empirische Informationen oder eine „Weltkenntnis“ liefern sollte, die für ihre Sozialisierung und Integration ins Leben in der Gesellschaft erforderlich waren ${ }^{24}$. Das damit anvisierte Orientierungswissen schließt u.a. ein, dass wir nicht nur Produkte, sondern auch Produzenten unserer individuellen und sozialen Entwicklung sind. Das menschliche Zusammenleben wird laut Kant durch eine allgemeine ,ungesellige Geselligkeit' bedingt und beschränkt, aber zugleich auch vorangetrieben, so dass wir hinsichtlich der sozialen Entwicklung mit anderen Menschen kooperieren und ihre Standpunkte begreifen müssen, um soziale Regeln oder eine funktionierende gesellschaftliche Welt zu schaffen. Daraus entstanden weitreichende philosophische Überlegungen und Positionen einschließlich einer anthropologisch basierten Theorie über die historische Dynamik der Menschheitsgeschichte, mit Konsequenzen für die nachfolgende Philosophie und Wissenschaft.

Eine spezielle sozialwissenschaftliche Disziplin im heutigen Sinne ist zwar bei Kant nicht zu finden; lediglich die Geschichtsschreibung unterscheidet er von seiner umfassenden Anthropologie, verbindet sie aber zugleich konzeptionell, methodologisch und thematisch eng mit ihr ${ }^{25}$. Seine Gedanken zu Geist, Gesellschaft und Geschichte sind sonst über verschiedene Schriften und Vorlesungen verteilt. Den Kern und das Schwerkraftzentrum seiner relevanten Arbeiten stellt vielmehr die ,pragmatische Anthropologie' dar, als eine empirische Disziplin, die Kant mehr als zwanzig Jahre lang lehrte. Kants Anthropologie stellt einen einzigartigen Versuch dar, eine Wissenschaft vom

\footnotetext{
${ }^{24}$ Vgl. Kant an Marcus Herz, Ende 1773, in: AA, Bd. X, 145-146.

${ }^{25}$ Mehr dazu in Sturm, Kant und die Wissenschaften vom Menschen, 341 et passim.
} 
Menschen zu schaffen, die verschiedene Aspekte der Zivilisierung, Kultivierung und Moralisierung des Menschen systematisch untersucht. Diese Wissenschaft sollte auch das gesellschaftliche Zusammenleben, also das Gebiet der modernen Sozialwissenschaften, die in differenzierter Form zu Kants Zeit nicht existierten, einschließen.

Das in den empirischen Humanwissenschaften gesammelte Wissen über den Menschen ist nach Kant sprunghaft angewachsen. Der Fundus der relevanten Disziplinen hat sich gegenüber der Situation, die Kant vorfand, stark verändert und in viele einzelne Disziplinen ausdifferenziert. Von ihren methodologischen, begrifflichen und ontologischen Voraussetzungen her sind diese Disziplinen äußerst heterogen. Obwohl einzelne Beispiele und Argumente Kants infolge dieser Entwicklungen obsolet wurden, gilt das nicht für die meisten wichtigsten politischen und sozialen Gehalte und Grundkonzepte seiner Philosophie, die in enger Beziehung zu mehreren Theorien und Schulen der Soziologie stehen. Kants Anthropologie, mit ihrem Konzept der pragmatischen Vernunft, spielt in diesem Kontext eine bedeutsame, wenn auch noch nicht völlig anerkannte Rolle.

\section{Kant und die Soziologie des 20. Jahrhunderts}

Aus gegenwärtiger Perspektive erscheint die These von Kants breitem Einfluss auf die Soziologie beinahe trivial. Insbesondere in makrosoziologischen Theorien aus der Mitte des 20. Jahrhunderts, etwa im strukturellen Funktionalismus von Talcott Parsons, waren Bezüge auf Kant - va. auf seine Epistemologie, auf sein Verständnis der Teleologie sowie auf seine Kritik am Utilitarismus - von grundlegender Bedeutung. Kant war präsent sowohl in der Soziologie der ersten Hälfte des 20. Jahrhunderts (u.a. bei Simmel und Tönnies, aber auch - trotz der Seltenheit direkter Erwähnungen - bei Max Weber) als auch in der Soziologie der Nachkriegszeit, etwa in den Ansätzen des Kritischen Rationalismus (v.a. bei Hans Albert), der Leipziger Schule, u.a. in Helmut Schelskys transzendentaler Gesellschaftstheorie, sowie der Kölner Schule (besonders prominent bei ihrem Gründer René König). Bezüge auf Kant wurden zum festen Bestandteil der Diskussionen um die Normativität 
als soziales ,Urphänomen' (König und Nachfolger), um die Methodologie und Grenzen des Kritischen Rationalismus ${ }^{26}$ und um die Form(en) der Erfahrung der Gesellschaft (Simmel u.a. ${ }^{27}$ ). Auch in den klassischen Studien zu den Ursprüngen und zur Entwicklung des soziologischen Denkens wird Kant als Denker anerkannt, bei dem die in der englischen und französischen Tradition entfaltenden Ansätze „zu einer neuen Stufe“ weiterentwickelt werden $^{28}$. Zu Kants Verdiensten werden v.a. die Anerkennung der zweifachen sozialen Natur des Menschen (als Bedürfnis- und Vernunftwesens), die Untersuchung der Verbindung zwischen Freiheit und Vergesellschaftung (und die daraus abgeleitete Revision von Rousseaus Theorie des Gesellschaftsvertrags) sowie die Trennung von Instinkt und Vernunft auf der anthropologischen Ebene gerechnet.

Wesentlich anders sieht es aber mit der Rolle von Kants anthropologischen Ideen in den Sozial- und Gesellschaftstheorien aus. Abgesehen von einzelnen Hinweisen in den historisch-soziologischen Studien, haben dieseIdeen für eine lange Zeit eine eher marginale Rolle gespielt - nicht zuletzt deswegen, weil die soziologische Relevanz von Kants Anthropologie sogar in den prominenten Studien sozialphilosophisch orientierter Denker (etwa bei Foucault $^{29}$ ) nicht thematisiert wurde. Im deutschsprachigen Raum wurde die anthropologisch orientierte Rezeption im Lichte konkurrierender Perspektiven, insbesondere derjenigen von Hegel und Marx, wie auch im Kontext der Konkurrenz zwischen Philosophischer Anthropologie und Kritischer Theorie begrenzt. Zumindest seit der Gründung der Frankfurter Schule hat sich insbesondere in der deutschen Soziologie die auf Horkheimer (und ursprünglich auf Marx) zurückgehende These verbreitet, dass Kants Philosophie der Individualität erst durch Hegel zur Sozialphilosophie weiterentwickelt bzw.

${ }^{26}$ Vgl. die Bezeichnung Kants als „geistigen Ahnen“ dieser Richtung in Georg Lührs, Thilo Sarrazin, Frithjof Spreer, Manfred Tietzel, „Kritischer Rationalismus und Sozialdemokratie“, in: Kritischer Rationalismus und Sozialdemokratie, ed. G. Lührs, T. Sarrazin, F. Spreer, M. Tietzel (Berlin/Bonn/Bad Godesberg: Dietz Verlag, 1975), 2.

${ }^{27}$ Spezifisch zu Kants Rolle in dieser Debatte s. Jürgen Schmitt, Die Grenzen der Rational Choice Theorie (Wiesbaden: Springer, 2000), $25 \mathrm{ff}$.

${ }^{28}$ S. Friedrich Jonas, Geschichte der Soziologie, Bd. 1: Aufklärung, Liberalismus, Idealismus, 2. Aufl. (Opladen: Westdeutscher Verlag, 1981), 123-135.

${ }^{29}$ Michel Foucault, Anthropologie du point de vue pragmatique. Introduction à l'Anthropologie [1964] (Paris: Vrin, 2008). 
vollendet wurde. In der englischsprachigen Soziologie wurde ihre Richtung hingegen im Wesentlichen durch die spezifische Deutung des Kantischen Begriffes des Pragmatischen im amerikanischen Pragmatismus geprägt.

Im Allgemeinen wurde die soziologische Rezeption von Kants anthropologischen Ideen wenigstens bis zur Mitte der 1990er Jahre durch mindestens drei Faktoren behindert. Die Grundlage für den ersten Faktor bildet eine Reihe von Vorstellungen und Thesen in der philosophischen und soziologischen Forschung, die Kants Anthropologie sowohl theoretisch-methodologisch als auch empirisch für obsolet erachten. Eine immer wiederkehrende Kritik, die an einzelnen Aspekten von Kants Anthropologie geäußert wird, betrifft ihren angeblichen Universalismus, Eurozentrismus sowie den veralteten Charakter vieler empirischer Beispiele und Schlussfolgerungen. Kants angebliche Thematisierung anthropologischer Fragen im eurozentrischen, z.T. auch kolonialistischen Kontext (in seinen Anthropologie-Vorlesungen wie Menschenkunde $e^{30}$ und in seinen kleinen Schriften der 1770-1780er Jahre), in dem Kant eine Rangordnung der Rassen nach der Anzahl ihrer Triebfedern ausarbeitet, wird sowohl innerhalb als auch außerhalb der Kantforschung einer scharfen Kritik unterzogen ${ }^{31}$. Die Kritik an Kants Beobachtungen zu den sozialen Geschlechterrollen ist sowohl in der Philosophie als auch in der Soziologie längst zu einem locuscommunis geworden. In diesem Lichte wird einerseits die Komplexität einzelner Fragen (wie diejenige nach den Begriffen ,Rasse und ,Volk` bei Kant) oft unterschätzt, andererseits die aus aktueller Perspektive anschlussfähigen Aspekte seiner Anthropologie außer Acht gelassen. Der zweite Faktor ist mit der Vorstellung verbunden, dass die wichtigsten Elemente von Kants Philosophie für die neuere Soziologie, die sich methodologisch zu reformieren und ihren Bezug zu empirischem Wissen neu zu deuten sucht, entweder überhaupt keinen Nutzen bringen

${ }^{30}$ Kant, VA, in: AA, Bd.XXV, insbes. 1187-1188.

${ }^{31}$ S. etwa Paul Gilroy, Against Race: Imagining Political Culture Beyond the Color Line (Cambridge: Belknap Press, 2000), 46. Vgl. die entgegengesetzten Positionen der Kantforscher Robert Bernasconi und Pauline Kleingeld: Robert Bernasconi, "Kant as an Unfamiliar Source of Racism", in: Philosophers on Race, ed. T. Lott, J. Ward (Oxford: Blackwell Publishing), 145-166; Pauline Kleingeld, "Kant's Second Thoughts on Colonialism”, in: Kant and Colonialism: Historical and Critical Perspectives, ed. K. Flikschuh, L. Ypi (Oxford: Oxford University Press, 2014), 46 ff. Kleingeld vertritt die These, dass bei Kant Mitte der 1790er-Jahre eine Abkehr von kolonialistischer Rassenhierarchie erfolgt. 
oder sogar schädlich sein können (vgl. dazu 2.1). Eine rare Ausnahme im deutschen Sprachraum bilden die Untersuchungen von Bernhard Schäfers, der in Kants Erkenntnistheorie und in seiner Idee der praktischen Vernunft die Grundlage für die Entwicklung einer, aufgeklärten', auf anthropologische Erkenntnisse Rücksicht nehmenden Sozialwissenschaft sieht ${ }^{32}$. Der dritte, historisch-philosophische Faktor ist v.a. spezifisch für den englischsprachigen Bereich. Nach dem Ende der ersten Welle des Pragmatismus und infolge des wachsenden Einflusses der analytischen Philosophie auf die weitere Entwicklung dieser Denkrichtung ist Kants Beitrag zur Formulierung des pragmatischen Programms in der Philosophie und in den Sozialwissenschaften im Allgemeinen schnell in Vergessenheit geraten.

Zwar hat sich die allgemeine Situation in den letzten zwei Jahrzehnten im Lichte der großen lebenswissenschaftlichen Entwicklungen zu ändern begonnen, indem viele sozialwissenschaftliche Projekte nicht nur anthropologische Ideen und Begriffe einbeziehen, sondern sich wieder an bestimmten Anthropologien orientieren, wie es für die deutsche Nachkriegssoziologie der Fall war, welche der damaligen Philosophischen Anthropologie nahestand $^{33}$. So schließt das Konzept einer reflexiven Anthropologie von Gesa Lindemann $^{34}$ sowie die von Robert Seyfert und Heike Delitz begründete ,Lebenssoziologie' an Helmuth Plessner an, wobei Plessner selbst anthropologische Themen (wie etwa die Rolle des ,moralischen Scheins', die Kant in der $A p H$ untersucht) und Begriffe Kants (z.B. ,ungesellige Geselligkeit') im Rahmen des eigenen soziologisch-anthropologischen Ansatzes instrumentalisiert. Dennoch bleiben Bezüge auf Kants anthropologische Ideen aus unterschiedlichen Gründen vergleichsweise spärlich, obwohl seine Anthropologie bei näherem Hinblick thematisch und methodologisch viel für aktuelle soziologische Studien und Theorien zu bieten hat.

${ }^{32}$ Bernhard Schäfers, „Kant und die Entwicklung einer aufgeklärten Erkenntnistheorie und Sozialwissenschaft", in: Bernhard Schäfers, Soziologie und Gesellschaftsentwicklung: Aufsätze 1966-1996 (Wiesbaden: Springer, 1996), 36-43.

${ }^{33}$ Vgl. dazu Hauke Brunkhorst, „Die Kommunikative Wende der Soziologie. Jürgen Habermas im Kontext der Nachkriegssoziologie“, in: Das Feld der Frankfurter Kultur- und Sozialwissenschaften nach 1945, ed. R. Faber (Würzburg: Königshausen \& Neumann, 2008), 167 ff.

${ }^{34}$ Gesa Lindemann, Die Grenzen des Sozialen: Zur sozio-technischen Konstruktion von Leben und Tod in der Intensivmedizin (München: Wilhelm Fink, 2002). 


\section{Schwerpunkte der soziologischen Rezeption von Kants Anthropologie}

Trotz ihres relativ fragmentarischen Charakters wäre es unmöglich, die ganze soziologische Rezeption von Kants anthropologisch orientierten Fragestellungen in einem kurzen Artikel zusammenzufassen. Im Folgenden werde ich sie an drei Beispielen - demjenigen des Pragmatismus, des Kritischen Rationalismus und der Philosophischen Anthropologie - knapp illustrieren.

Im ,alten' amerikanischen Pragmatismus vor der analytischen Wende hat Kants Anthropologie v.a. auf methodologischer Ebene eine beträchtliche Rolle gespielt und dadurch die durch das philosophische Programm des Pragmatismus beeinflussten Bereiche der Sozialwissenschaften betroffen. Zwar spielte Kants Begriff des Pragmatischen bei den Gründern des Pragmatismus nicht die gleiche Rolle: während Ch. S. Peirce in Kants pragmatischer Anthropologie eine Art praktischer Ethik sah, die die Erreichung ihrer Zwecke mithilfe des experimentellen Ansatzes anvisiert und somit im Einklang mit seinem eigenen pragmatischen Projekt steht, hat sich Willam James nur gelegentlich auf Kant berufen, v.a. dort, wo es um die pragmatische Rolle der menschlichen Wohlfahrt geht. Entsprechend gab es auch keine Eindeutigkeit oder langfristige Kontinuität in der pragmatisch orientierten soziologischen Rezeption.

Selbst die Genese des Begriffs 'Pragmatismus' hat eine direkte Verbindung zu Kants Anthropologie. Peirces Verwendung des Begriffs geht zurück auf seine Kant-Lektüren der 1860-1870er Jahre sowie auf seine scharfe Unterscheidung zwischen , pragmatisch' und ,praktisch' bei Kant. Wegen seiner Relation zu apriorischen Bedingungen der Erfahrung erwies sich das kantische Konzept des Praktischen als nutzlos für die Beschreibung seines Ansatzes. Als ,Weltkenntnis' bietet das Pragmatische hingegen eine empirische Verortung für ihn. In einer nachgelassenen Schrift von Peirce aus dem Jahre 1905, die erst 50 Jahre später zusammen mit anderen "Selected Writings" veröffentlicht wurde, finden wir folgende Passage, die die Grundlagen dieser Unterscheidung näher erklärt „[...] for one who has learned philosophy out of Kant [...] praktisch and pragmatisch were as far apart as the two poles, the former 
belonging in a region of thought where no mind of the experimentalist type can ever make sure of solid ground under his feet, the latter expressing relation to some definite human purpose ${ }^{\text {"35 }}$. Obwohl Peirce die Distanz zwischen dem Pragmatischen und dem Praktischen auf jeden Fall deutlich übertreibt, insofern das Pragmatische bei Kant ganz deutlich zur Dimension des Praktischen gehört,hat er bestimmt Recht darin, dass sein eigener Ansatz aus Kants Perspektive vielmehr, pragmatisch' als , praktisch erscheint $^{36}$.

Insofern die Untersuchungen zur Geschichte des amerikanischen Pragmatismus in vielerlei Hinsicht noch lückenhaft bleiben, gilt das auch für den Einzelfall der anthropologisch orientierten Kant-Rezeption. Hinsichtlich des Teilgebiets der pragmatischen Soziologie können wir dennoch mit Sicherheit feststellen, dass Kants Anthropologie einen Einfluss auf George Herbert Mead ausgeübt hat. Für Mead war Kant immer ein kritischer Bezugspunkt. Bereits in seinem nicht beendeten Dissertationsprojekt, das der Kritik an einer empiristischen Konzeption des Raumes gewidmet war, versuchte er seine eigene Idee des Raums als Kooperation von Hand und Auge in Auseinandersetzung mit Kants Phänomenalismus zu entwickeln ${ }^{37}$. Auch sein Konzept des Selbst wollte Mead womöglich von Kant explizit distanzieren. So beschreibt er in seinem frühen Artikel Psychology of Punitive Justice (1918) die kantische Reaktion des Selbst „auf abstrakte Universalien“ als einen fundamentalen Irrtum der Argumentation, weil der „Weg aus der Selbstsucht“ vielmehr „über die Anerkennung des genuin sozialen Charakters der menschlichen Natur“ verlaufe $^{38}$. Doch gerade der letztere Mangel lässt sich durch den Bezug auf Kants pragmatische Anthropologie im Wesentlichen beseitigen. Bei einem näheren Blick sehen wir, dass Meads Theorie des sozialen Selbst die anthropologisch-pragmatischen Aspekte von Kants Theorie der menschlichen Erfahrung, die u.a. durch seinen Begriff der , ungeselligen Geselligkeit‘ zur

${ }^{35}$ Charles S. Peirce, Collected Papers, vol. 5, ed. C. Hartshorne, P. Weiss (Cambridge: Harvard University Press, 1934), 412.

${ }^{36}$ Vgl. dazu auch Marcus Willaschek, "Kant and Peirce on Belief”, in: Pragmatism, Kant and Transcendental Philosophy, ed. G. Gava, R. Stern (New York: Routledge, 2015), 133-151.

${ }^{37}$ S. dazu Hans Joas, Praktische Intersubjektivität: Die Entwicklung des Werkes von George Herbert Mead (Frankfurt a.M.: Suhrkamp, 1989), 25.

${ }^{38}$ George H. Mead, Gesammelte Aufsätze, Bd. 1, ed. Hans Joas (Frankfurt a.M.: Suhrkamp, 1980), 262. 
Erscheinung kommen, übernimmt. Meads allgemeine Darstellung der Beziehungen zwischen dem Selbst und dem generalisierten Anderen bzw. dem sozialen Ganzen in Meads wohl bekanntester Schrift Mind, self and society (1934), die nach seinem Tode zusammengesetzt und veröffentlicht wurde, hat deutliche Parallelen zu Kants Erklärung der Interaktion zwischen dem Individuum und der Gesellschaft, u.a. in seinen Vorlesungen zur Anthropo$\operatorname{logie}{ }^{39}$. Indem Mead, gleich anderen Pragmatisten, sich gegen die kantischen Universalien und somit gegen die Vorstellung vom privilegierten Charakter der Formen im Vergleich zu Objekten wendet, beschreibt er, gleich Kant, die Genese und Struktur der sozialen Prozesse durch die Optik des Individuellen. Insofern können wir - trotz des zweideutigen, komplexen Charakters der pragmatistischen Kant-Rezeption - auch allgemeiner vom teilweise positiven Einfluss der Anthropologie Kants auf den soziologischen Ansatz des symbolischen Interaktionismus sprechen.

Als Urvater des Kritischen Rationalismus wird Kant wenigstens seit Karl Poppers Gedächtnisrede Immanuel Kant: the Philosopher of the Enlightenment (1954) gekrönt. Auf erkenntnistheoretischer Ebene setzt der Kritische Rationalismus genau dort an, wo die „Kopernikanische Wende“ Kants anfängt d.h. bei der Kritik am Empirismus und Dogmatismus. Dabei orientiert er sich an Kants Konzept der Vernunft, des Urteilens und der Aufklärung, im Sinne der Suche nach rationalen Lösungen sozialer Probleme. Auf ethischer Ebene wird vor allem Kants Bild des Menschen als Gesetzgebers der Moral positiv rezipiert ${ }^{40}$. Dass ein wichtiger Teil dieser Rezeption anthropologisch orientiert ist, bleibt in der Forschung allerdings - zu Unrecht - weitgehend unbeachtet. Nur wenige Autoren, wie etwa der deutsche Soziologe Bernard Schäfers, deuten auf die zentrale Rolle von Kants pragmatischem Denken für die methodologische Selbstaufklärung moderner Sozialwissenschaften - und spezifisch des Kritischen Rationalismus - hin. Von Bedeutung sind in diesem Lichte die Kriterien der Wissenschaftlichkeit hinsichtlich der Anthropologie

${ }^{39}$ Vgl. etwa I. Kant, VA-Mrongovius, in: AA, Bd. XXV, 1416.

${ }^{40}$ Dass diese Rezeption nicht vollständig unkritisch ist, wird ebenfalls v.a. bei Popper explizit betont. So werden das Konzept der synthetischen Urteile a priori, die Vorstellung von den uns gemeinsamen Norm-Anschauungen und die Kantische Idee der Zeit im Kritischen Rationalismus nicht akzeptiert. Vgl. dazu auch Schäfers, „Kant und die Entwicklung einer aufgeklärten Erkenntnistheorie und Sozialwissenschaft", 30. 
Kants sowie sein Fallibilismus und seine anthropologisch geprägten Aussagen über die gesellschaftlichen Aspekte des menschlichen Lebens.

Ironischerweise wird eine detaillierte Analyse der anthropologischen Kant-Rezeption bei den Anhängern des Kritischen Rationalismus dadurch erschwert, dass viele von ihnen (mit der Ausnahme von Popper) ihre impliziten Bezüge auf Kant und den Kantianismus als evident und daher als nicht begründungsbedürftig betrachteten ${ }^{41}$. Das ist auch bei Hans Albert der Fall, der als Hauptvertreter dieser soziologischen Richtung gilt. Trotz des oft spürbaren Mangels an konkreten Zitaten und Bezügen lässt sich die methodologische und thematische Abhängigkeit relativ leicht demonstrieren. So lässt sich ein wesentlicher Anteil des von Albert ${ }^{42}$ und vielen anderen vertretenen Fallibilismus, der auf den begrenzten Charakter der Urteilsfähigkeit menschlicher Vernunft Rücksicht nimmt, von Kants Vorstellung vom Menschen als nicht völlig vernünftigem, aber vernunftfähigem Wesen ableiten. Auch Kants Idee, dass Menschen ihr Vermögen pragmatisch gebrauchen sollen, zusammen mit der Vorstellung von Aufklärung als unaufhörlicher kritischer Beseitigung von Vorurteilen, steht im Einklang mit dem für den Kritischen Rationalismus grundlegenden Prinzip der handlungsorientierten Problemlösung.Die Forderung nach Freiheit sowie die Verbindung zwischen Freiheit und Selbstverwirklichung im Handeln, die im Kritischen Rationalismus eine zentrale Stellung hat, sieht ihrerseits als praktische Weiterentwicklung von Kants Idee des Menschen als frei handelnden Wesens in $\mathrm{ApH}$ aus.

Eine der interessantesten Kapitel der soziologischen Rezeption von Kants Anthropologie betrifft wiederum den Begriff der ungeselligen Geselligkeit und illustriert die Konkurrenz zwischen zwei anthropologischen Perspektiven - denjenigen von Kant und von Helmuth Plessner. Plessner, der zusammen mit Max Scheler die Philosophische Anthropologie - nunmehr als eine philosophische Denkrichtung - neu gründete, ist zugleich durch seine philosophischen und soziologischen Leistungen bekannt. Letztere bestehen primär in der Ausarbeitung eines Konzepts der Gesellschaft im Kontrast zu zwei

${ }^{41}$ S. wiederum Schäfers, „Kant und die Entwicklung einer aufgeklärten Erkenntnistheorie und Sozialwissenschaft", 30-31.

${ }^{42}$ S. insbes. Alberts Traktat über Kritische Vernunft (1968), Kritische Vernunft und menschliche Praxis (1977), Die Wissenschaft und die Fehlbarkeit der Vernunft (1982) sowie Freiheit und Ordnung (1986). 
Hauptformen der Gemeinschaft (s. Grenzen der Gemeinschaft, 1924) sowie in der Verbindung zwischen Biologie, die er in seinem programmatischen Werk Die Stufen des Organischen und der Mensch (1928) sowie in späteren Schriften (Macht und menschliche Natur, Lachen und Weinen u.a.) darstellte und weiterentwickelte. Seine Kantrezeption war zum Teil durch den Neukantianismus vermittelt. Dennoch bezieht er sich auch sehr oft direkt auf Kant, sei es in einem positiven Kontext (z.B. wenn es um die Struktur menschlicher Erfahrung geht) oder vom Standpunkt der Kritik am Universalismus und ,Europäismus' der ,klassischen' Anthropologien Kants und Hegels. Ein wesentlicher Teil moderner deutscher Soziologie, repräsentiert z.B. durch die Schriften von Gesa Lindemann, stützt sich auf Plessners Anthropologie, wobei Plessners anthropologische Ideen auch die dritte Welle der deutschen Kritischen Theorie, v.a. Axel Honneth, beeinflusst haben.

In seiner Schrift „Ungesellige Geselligkeit: Anmerkungen zu einem Kantischen Begriff (1966) kritisiert Plessner die in der Idee zu einer allgemeinen Geschichte in weltbürgerlicher Absicht formulierte und in der Anthropologie in pragmatischer Hinsicht wiederholte These, laut der die Menschen als Individuen egoistisch sind, weil sie dazu durch „Ehrsucht, Herrschsucht oder Habsucht" getrieben werden. Es ist Plessners zentrales Anliegen, die anthropologische Rolle des menschlichen Propriums, die Kant der Idee des natürlichen Hangs zur Vergesellschaftung im Sinne des Widerstands dem egoistischen Hang zuordnet, in Frage zu stellen. Plessner wirft Kant vor, dass Letzterer den soziologisch basalen, in Plessners Gemeinschaft-Schrift mehrfach betonten Unterschied zwischen individuellem und sozial bedingtem Handeln (also zwischen Individuum und Person mit einer sozialen Maske) missachtet. Plessner zufolge gerät Kant damit in die Falle von pars pro toto, indem er die Schlussfolgerungen über das soziale Benehmen der Menschen auf ihre individuellen, natürlichen Eigenschaften erweitert. Zugleich sind auch Herrschsucht sowie insbesondere Ehrsucht und Habsucht aus Plessners Sicht keinesfalls als menschliche Konstanten zu verstehen, insofern solche Annahme auf einem starken Anthropomorphismus beruht, der den empirischen Ergebnissen der Zoologie widerspricht, und andererseits auch die Unterschiede zwischen Gesellschaftsformen nicht berücksichtigt. Insofern sei die 'ungesellige Geselligkeit' zwar ein mögliches Element rationaler Auffassung gesellschaftlicher Prozesse, nicht aber die anthropologische Grundlage 
dieser Auffassung ${ }^{43}$. Ob diese Kritik Kant völlig gerecht wird, lässt sich allerdings bestreiten, v.a. weil sie trotz einiger Korrekturen den Kern der Kantischen Argumentation nicht widerlegt, laut dem , ungesellige Geselligkeit' in der Spannung zwischen dem natürlichen Hang zur Vergesellschaftung und den egoistischen Motiven jedes Individuums besteht. Obwohl der Begriff der ungeselligen Geselligkeit zu allgemein wirken mag und sowohl aus Plessners Sicht als auch aus der Sicht mancher Soziologen keinen Anschluss an konkrete empirische Beispiele hat, wäre er gerade für die neueren soziologischen Ansätze, etwa auf Spuren von Ralf Dahrendorfs Konfliktsoziologie, von primärer Bedeutung, nicht zuletzt im Rahmen der andauernden Kommunitarismusdebatte. Da Kant, anders als Hegel, das menschliche Streben zur sozialen Anerkennung auch aus anthropologischer Perspektive thematisiert ${ }^{44}$, bietet sein Ansatz in diesem Kontext eine Alternative zur konfliktsoziologischen Methode der Frankfurter Schule, die sich primär auf Hegels Sozialphilosophie sowie auf Plessners philosophische Anthropologie bezieht ${ }^{45}$.

Die Aufdeckung impliziter und nicht selten fragmentarischer Aspekte der deutsch- und englischsprachigen soziologischen Rezeption von Kants Anthropologie im 20. Jahrhundert ist nicht nur eine rein historisch orientierte, systematisierende Aufgabe. Eine weitgehendere Untersuchung kann die Fragen nach der Diskrepanz zwischen Kants Verständnis des Pragmatischen und demjenigen der Soziologie wie auch nach den Vorteilen und Defiziten von Kants Anthropologie aus soziologischer Sicht, im Vergleich zum Ansatz Hegels, beantworten und somit - chronologisch und inhaltlich - eine Brücke zur Problematik aktueller soziologischer Debatten schlagen. Es ist klar, dass die Optik von Kants Anthropologie eine potentielle Alternative zu anderen anthropologisch erfahrenen Perspektiven der modernen Soziologie bieten kann. Ob diese Alternative auch fruchtbarer ist, soll die Zukunft zeigen.

${ }^{43}$ Helmuth Plessner, „Ungesellige Geselligkeit. Anmerkungen zu einem Kantischen Begriff [1966]“, in: Helmuth Plessner, Gesammelte Schriften, Bd. VIII: Conditio humana (Frankfurt a.M.: Suhrkamp, 1983), 306.

${ }^{44}$ Vgl. Kant, VA, Bd. XXV, 12-13, 245 sowie Sturm, „Reines und empirisches Bewusstsein in Kants Anthropologie“, 214.

${ }^{45}$ S. Axel Honneth, Kampf um Anerkennung (Frankfurt a.M.: Suhrkamp Verlag, 1992). 


\section{Bibliographie}

Albert Hans. 1968. Traktat über Kritische Vernunft. Tübingen: Mohr.

Albert Hans. 1977. Kritische Vernunft und menschliche Praxis. Stuttgart: Reclam.

Albert Hans. 1982. Die Wissenschaft und die Fehlbarkeit der Vernunft. Tübingen: Mohr.

Albert Hans. 1986. Freiheit und Ordnung. Zwei Abhandlungen zum Problem einer offenen Gesellschaft. Tübingen: Mohr.

Bernasconi Robert. 2002. "Kant as an Unfamiliar Source of Racism”. In: Philosophers on Race. Ed. T. Lott, J. Ward. 145-166. Oxford: Blackwell Publishing.

Brandt Reinhardt, Werner Stark. 1997. "Einleitung”. In: Immanuel Kant. Gesammelte Schriften. Akademie-Ausgabe. Bd. XXV: Vorlesungen über Anthropologie, VII-CLI. Berlin: De Gruyter.

Brunkhorst Hauke. 2008. "Die Kommunikative Wende der Soziologie. Jürgen Habermas im Kontext der Nachkriegssoziologie". In: Das Feld der Frankfurter Kulturund Sozialwissenschaften nach 1945. Ed. R. Faber. 167-188. Würzburg: Königshausen \& Neumann.

Cohen Alix. 2009. Kant and the Human Sciences: Biology, Anthropology and History. New York: Palgrave Macmillan.

Dahrendorf Ralf. 1967. Pfade aus Utopia: Arbeiten zur Theorie und Methode der Soziologie. München: Piper.

Dahrendorf Ralf. 1972. Konflikt und Freiheit: auf dem Weg zur Dienstklassengesellschaft. München: Piper.

Foucault Michel. 2008. Anthropologie du point de vue pragmatique. Introduction à l'Anthropologie [1964]. Paris: Vrin.

Frierson Patrick J. 2003. Freedom and Anthropology in Kant's Moral Philosophy. Cambridge: Cambridge University Press.

Gilroy Paul. 2000. Against Race: Imagining Political Culture Beyond the Color Line. Cambridge: Belknap Press.

Guyer Paul. 2015. "Play and Society in Kant's Anthropology". In: Reading Kant's Lectures, ed. R. R. Clewis. 223-241. Berlin/Boston: De Gruyter.

Hanna Robert. 2006. Kant, Science, and Human Nature. Oxford/New York: Oxford University Press.

Hart Keith. 2010. "Kant, 'anthropology' and the new human universal”. Social Anthropology/Anthropologie Sociale 18, 4: 441-447.

Honneth Axel. 1992. Kampf um Anerkennung. Frankfurt a.M.: Suhrkamp Verlag.

Joas Hans. 1989. Praktische Intersubjektivität: Die Entwicklung des Werkes von George Herbert Mead. Frankfurt a.M.: Suhrkamp. 
Jonas Friedrich. 1981. Geschichte der Soziologie. Bd. 1: Aufklärung, Liberalismus, Idealismus. 2. Aufl. Opladen: Westdeutscher Verlag.

Kant Immanuel. 1900-. Gesammelte Schriften. Akademie-Ausgabe. Berlin.

Kleingeld Pauline. 2014. "Kant's Second Thoughts on Colonialism". In: Kant and Colonialism: Historical and Critical Perspectives. Ed. K. Flikschuh, L. Ypi. 43-67. Oxford: Oxford University Press.

Lindemann Gesa. 2002. Die Grenzen des Sozialen: Zur sozio-technischen Konstruktion von Leben und Tod in der Intensivmedizin. München: Wilhelm Fink.

Louden Robert B. 2000. Kant's Impure Ethics: From Rational Beings to Human Beings. New York: Oxford University Press.

Lührs Georg, Thilo Sarrazin, Frithjof Spreer, Manfred Tietzel. 1975. "Kritischer Rationalismus und Sozialdemokratie". In: Kritischer Rationalismus und Sozialdemokratie. Ed. G. Lührs, T. Sarrazin, F. Spreer, M. Tietzel. 1-54. Berlin/Bonn/Bad Godesberg: Dietz Verlag.

Mead George H. 1918. “The Psychology of Punitive Justice”. American Journal of Sociology 23: 577-602.

Mead George H. 1980. Gesammelte Aufsätze. Bd. 1. Ed. Hans Joas. Frankfurt a.M.: Suhrkamp.

Peirce Charles S. 1934. Collected Papers. Vol. 5. Ed. C. Hartshorne, P. Weiss. Cambridge: Harvard University Press.

Plessner Helmuth. 1983. "Ungesellige Geselligkeit. Anmerkungen zu einem Kantischen Begriff [1966]". In: Helmuth Plessner, Gesammelte Schriften. Bd. VIII: Conditio humana. 294-306. Frankfurt a.M.: Suhrkamp.

Popper Karl. 1996. "Immanuel Kant: the Philosopher of the Enlightenment". In: In Search of a Better World: Lectures and Essays from Thirty Years. 126-136. London/New York: Routledge.

Rossi Philip J. 2013. "Cosmopolitanism - Kant's Social Anthropology of Hope". In: Kant und die Philosophie in weltbürgerlicher Absicht: Akten des XI. Kant-Kongresses 2010. Bd. 4. Ed. S. Bacin, A. Ferrarin, C. La Rocca, M. Ruffing. 827-837. Berlin: De Gruyter.

Schäfers Bernhard. 1996. "Kant und die Entwicklung einer aufgeklärten Erkenntnistheorie und Sozialwissenschaft". In: Bernhard Schäfers. Soziologie und Gesellschaftsentwicklung: Aufsätze 1966-1996. 22-43. Wiesbaden: Springer.

Schmitt Jürgen. 2000. Die Grenzen der Rational Choice Theorie. Wiesbaden: Springer. Stark Werner. 2000. "Diese Dinge' und andere Varia”. In: Zustand und Zukunft der Akademie-Ausgabe von Kant's gesammelten Schriften. Kant-Studien. Bd. 91. Sonderheft. Ed. R. Brandt, W. Stark. 1-16. Berlin: De Gruyter.

Sturm Thomas. 2009. Kant und die Wissenschaften vom Menschen. Paderborn: Mentis Verlag. 
Sturm Thomas. 2017. "Reines und empirisches Bewusstsein in Kants Anthropologie: Das 'Ich' und die rationale Charakterentwicklung". In: Immanuel Kant - Die Einheit des Bewusstseins. Ed. G. Motta, U. Thiel. 195-220. Berlin/Boston: De Gruyter.

Waldenfels Bernhard. 2015. "Das Dilemma einer ungeselligen Geselligkeit". In: Bernhard Waldenfels. Sozialität und Alterität: Modi sozialer Erfahrung. 29-72. Berlin: Suhrkamp.

Willaschek Marcus. 2015. "Kant and Peirce on Belief". In: Pragmatism, Kant and Transcendental Philosophy. Ed. G. Gava, R. Stern. 133-151. New York: Routledge. Wilson Holly J. 2006. Kant's Pragmatic Anthropology: Its Origin, Meaning and Critic Significance. New York: SUNY Press.

Wood Allen. 1991. "Unsocial Sociability: The Anthropological Basis of Kantian Ethics”. Philosophical Topics 19: 325-351.

Wood Allen. 2003. "Kant and the Problem of Human Nature". In: Essays on Kant's Anthropology. Ed. B. Jacobs, P. Kain. 38-59. Cambridge: Cambridge University Press.

Zhavoronkov Alexey, Alexey Salikov. 2018. "The Concept of Race in Kant's Lectures on Anthropology". Con-Textos Kantianos 7: 275-292.

Zöller Günter. 2011. "Kant's Political Anthropology". Kant Yearbook 3: 131-161.

\section{Abstract \\ Social Aspects of Kant's Anthropology and Their Influence on the 20th-Century Sociology: Problems and Cases}

The paper examines the mostly neglected social aspects of Kant's anthropology, as a foreword to the discussion concerning the influence and the actuality of Kant's anthropology from the modern perspective of social sciences, and sociology in particular. Its first part, containing a brief description of the structure of Kant's pragmatic anthropology, analyzes its social elements, with an emphasis on the anthropological role of the concept of 'unsocial sociability'. In the second part, I shed light on the main factors which so far have been a major impediment to a wider sociological reception of Kant's ideas. In the third part, I address the question of Kant's influence on the European and American sociology - on the examples of George H. Mead's pragmatism, Hans Albert' critical rationalism and philosophical anthropology of Helmuth Plessner.

Keywords: Kant, anthropology, Mead, Plessner, Albert 